\title{
EXPERIMENTAÇÃO NUMÉRICA E FÍSICA DA EROSÃO EM CICLONES
}

\author{
T. A. SEDREZ ${ }^{1 *}$, R. K. DECKER ${ }^{1}$, D. NORILER ${ }^{1}$, H. F. MEIER ${ }^{1}$ \\ ${ }^{1}$ Universidade Regional de Blumenau, Departamento de Engenharia Química \\ e-mail: thisedrez@gmail.com
}

\begin{abstract}
RESUMO
A erosão é o desgaste mecânico na parede dos equipamentos por partículas transportadas por escoamento de fluido. Este fenômeno representa um grave problema nas unidades de FCC (craqueamento catalítico fluido), especialmente em ciclones, reduzindo a vida útil do equipamento e causando paradas não programadas. Assim, o objetivo deste trabalho é analisar a erosão causada pelo impacto das partículas de catalisador de FCC em uma geometria de ciclone otimizada para operar com altas vazões, mediante a realização de estudos experimentais conjugados com estudos de simulação numérica. O escoamento gás-sólido foi estudado por meio da abordagem euleriana-lagrangeana com o modelo de turbulência RSM-SSG (Reynolds Stress Model - Quadrático). A taxa de erosão foi calculada por dois modelos de erosão. Experimentações físicas foram realizadas para validar os resultados numéricos com ciclones construídos em gesso para acelerar o processo de erosão. Os resultados numéricos apresentaram boa concordância qualitativa com os dados experimentais, mostrando a região na parede do ciclone onde a erosão é mais evidente e, de forma quantitativa em alguns dos casos estudados.
\end{abstract}

\section{INTRODUÇÃO}

Ciclones são amplamente utilizados nas indústrias para a separação de partículas sólidas de uma corrente gasosa, por terem sua geometria simplificada, alta eficiência na coleta de partículas, e baixo custo de investimento e manutenção. No entanto, o contato das partículas sólidas com as paredes do ciclone causa erosão. A erosão vem se tornando um grande problema industrial, principalmente em escoamentos gás-sólidos, limitando o tempo de vida dos equipamentos e ocasionando paradas não programadas nas plantas industriais (UTIKAR et al., 2010).

Segundo Bitter (1963) o desgaste resultante do impacto das partículas de movimento livre em um sólido é definido como erosão, sendo esse desgaste puramente mecânico e observado em sistemas em que as partículas se movem a altas velocidades.

De acordo com a literatura, Finnie (1960) propôs a primeira derivação analítica de um modelo de corte erosivo para uma única partícula atingindo um material, discutindo algumas condições do escoamento que podem levar a erosão e analisando o mecanismo de remoção para materiais dúcteis e frágeis. A partir daí, outros modelos de erosão surgiram.

Encontram-se, na literatura, muitos estudos de erosão, tanto do ponto de vista experimental quanto numérico. A abordagem Euler-Lagrange é predominante e fatores como velocidade (MAZUMDER, 2012), taxa mássica, tamanho da partícula (DENG et al., 2013) e modelos de erosão (PEREIRA; SOUZA; MARTINS, 2014) são avaliados nos mais variados estudos e geometrias, como em 
cotovelos, dutos, tês, curvas, ciclones e calhas.

Assim, este trabalho tem como objetivo analisar a erosão em ciclones causada por partículas de FCC, tanto do ponto de vista experimental quanto numérico. Para tal se utilizou ciclones feitos em gesso na experimentação física para acelerar a erosão, possibilitando o cálculo da taxa de erosão e a observação dos locais erodidos (KRAXNER et al. (2013)). E, um dispositivo para estabilização do vórtice.

O estabilizador de vórtice é uma placa originalmente colocada a $1 / 3$ do final do cone do ciclone para estabilizar o vórtice central nessa região e, consequentemente, é uma alternativa para diminuir a erosão nesta área (CHEN et al. (2010)). A localização do estabilizador está relacionada ao fato de que a velocidade angular aumenta quando gás e sólido se movimentam da seção cilíndrica para a cônica. A intenção é de que o vórtice terminará na placa plana e que a intensidade de espirais de sólido abaixo da placa seja reduzida.

Para a simulação numérica, a abordagem Euler-Lagrange juntamente com o modelo de turbulência RSM e os modelos de erosão geral (DET NORSKE VERITAS, 2007) e de Oka, Okamura e Yoshida (2005) e Oka e Yoshida (2005) foram utilizados no software comercial FLUENT 14.0 (ANSYS, 2011) para estudos em um ciclone otimizado por Sgrott et al. (2015). O efeito da velocidade de entrada do gás no ciclone foi avaliado para uma taxa mássica de sólidos fixa tanto do ponto de vista experimental quanto numérico.

\section{MATERIAIS E MÉTODOS}

Os materiais e métodos utilizados nas experimentações física e numérica estão apresentados a seguir.

\subsection{Experimentação Física}

Os experimentos físicos deste trabalho foram realizados na Unidade Experimental de Dutos e Ciclones (UE-DC), apresentada na Figura 1. Na unidade, um tubo de Pitot (02) é conectado a um sensor diferencial de pressão para medir a velocidade média de entrada do gás; um exaustor (09) mantém a velocidade constante. A fase sólida é carregada no sistema através de um alimentador de sólidos (06). As partículas são separadas por meio de um ciclone (08) e um filtro de mangas (11). O detalhamento do ciclone Sgrott_103-500 está apresentado pela Tabela 1, acompanhado da Figura 2, sendo a espessura da parede de gesso de $10 \mathrm{~mm}$. Os ciclones confeccionados em gesso foram feitos em partes, conforme mostra a Figura 3 e, os moldes impressos em

Figura 1 - Representação ilustrativa da Unidade Experimental de Dutos e Ciclones (UE-DC).

01 - Entrada de ar

02 - Tubo de Pitot

03 - Sensor de pressão

04 - Sensor de pressão

05 - Sensor de temperatura

06 - Alimentador de sólidos

07 - Sensor de pressão

08 - Ciclone de gesso

09 - Exaustor

10 - Caixa coletora

11 - Filtro de mangas

12 - Computador e painel de controle

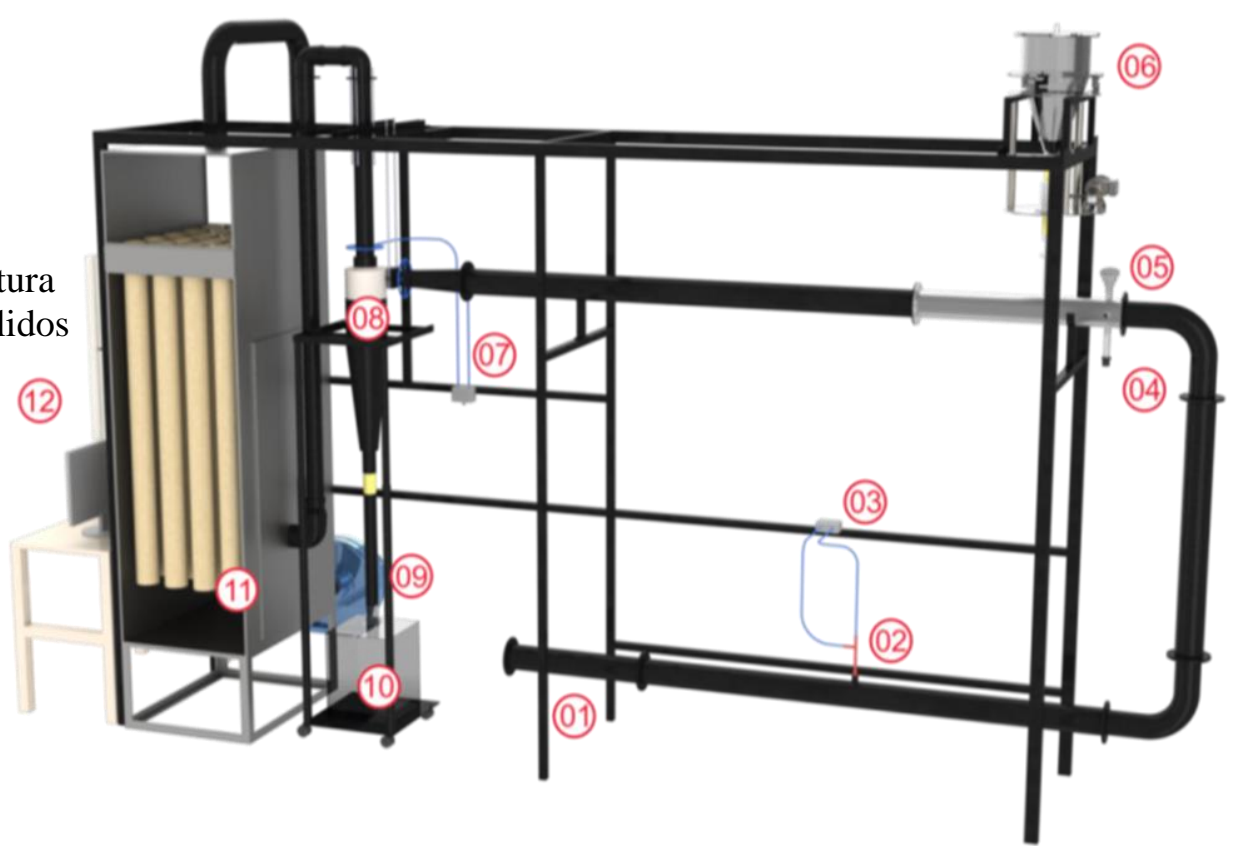




\section{ENEMP \\ CONGRESSO BRASILEIRO \\ DE SISTEMAS PARTICULADOS \\ São Carlos - SP}

Tabela 1 - Detalhamento do ciclone Sgrott_103500.

\begin{tabular}{ll}
\hline Relações Geométricas & \multicolumn{1}{c}{ Dimensões $(\mathrm{mm})$} \\
\hline Ds & 73,8 \\
Dc & 150 \\
Dl & 54,9 \\
Le & 60,3 \\
Ls & 111,6 \\
Lc & 210,6 \\
Lco & 441,3 \\
b & 30,9 \\
\hline
\end{tabular}

Figura 2 - Ciclone Sgrott_103-500.

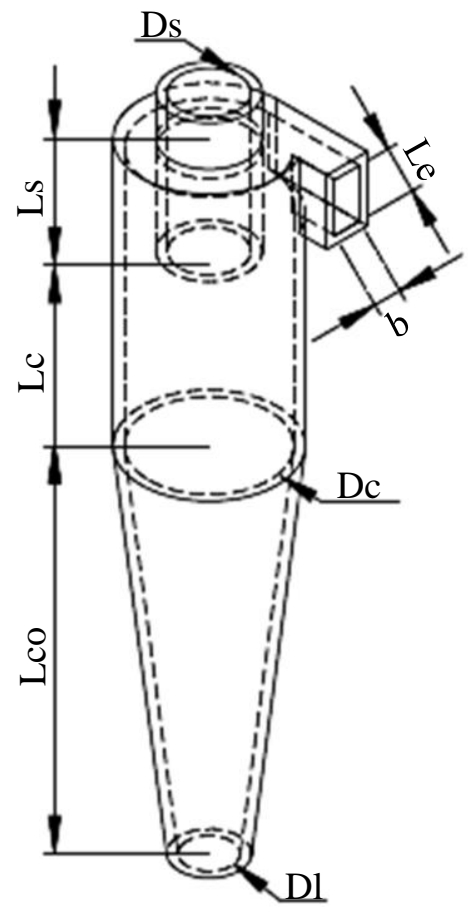

uma impressora 3D. O ciclone montado é apresentado na Figura 4. Na experimentação física, o estabilizador de vórtice também foi utilizado, a 1/3 do final do cone (Figura 5). Esta peça é feita de aço inoxidável e sua área de passagem é ligeiramente maior que a área da saída do cone.

A taxa mássica de sólidos foi mantida constante $(11,8 \mathrm{~g} / \mathrm{s})$ e a velocidade de gás na entrada do ciclone foi variada $(25,30$ e 35 $\mathrm{m} / \mathrm{s}$ ) em três baterias de experimentos. O Grupo 1 não utilizou estabilizador de vórtice e colocou-se um reforço estrutural ao redor da
XXXVII ENEMP

Figura 3 - Moldes e peças em gesso. a) tampas; b) duto de saída; c) entrada; d) cilindro e e) cone.

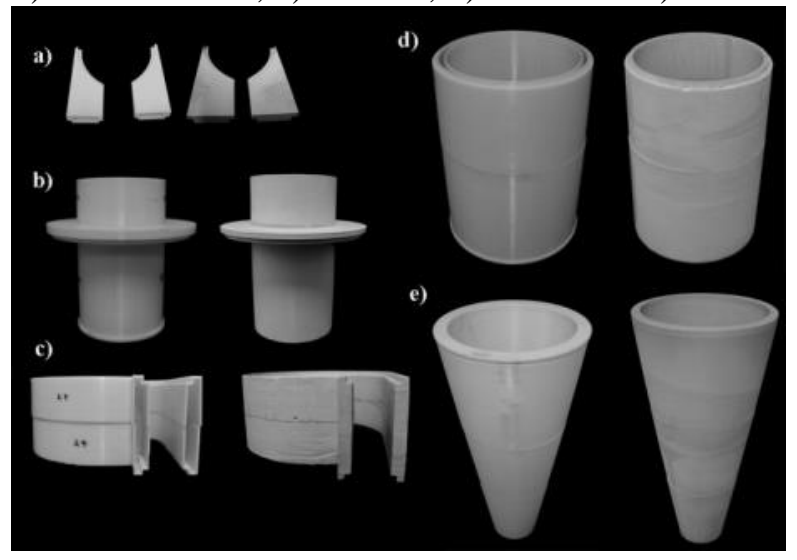

Figura 4 - Ciclone em gesso.

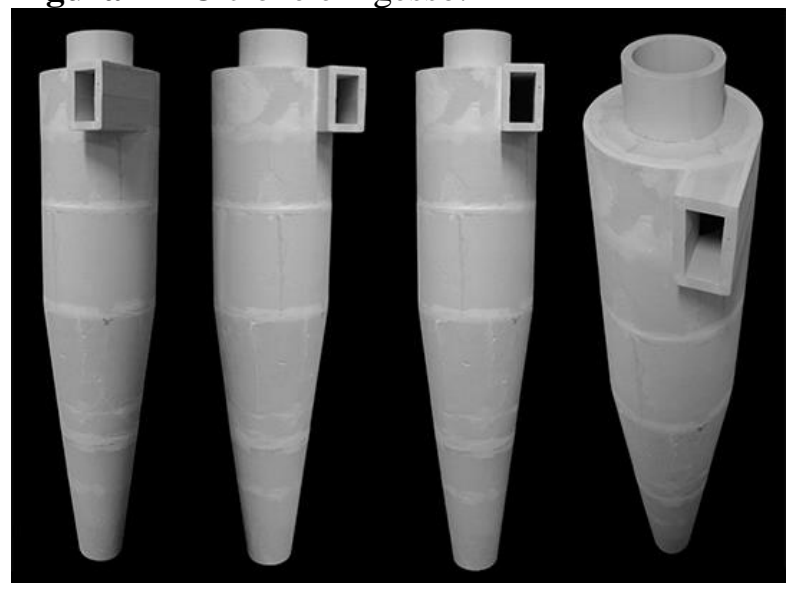

Figura 5 - Estabilizador de vórtice.
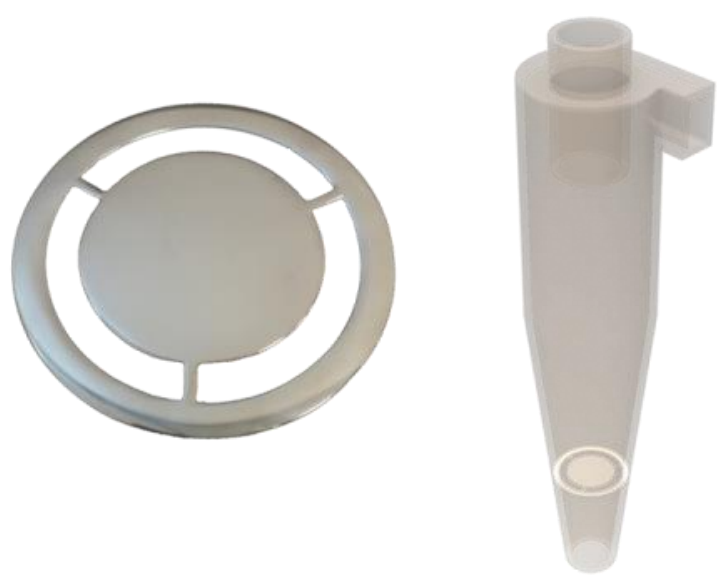

entrada do ciclone para possibilitar maior erosão no corpo do ciclone; o Grupo 2 foi idêntico ao Grupo 1, porém utilizou o 
Figura 6 - Distribuição granulométrica das partículas de FCC.

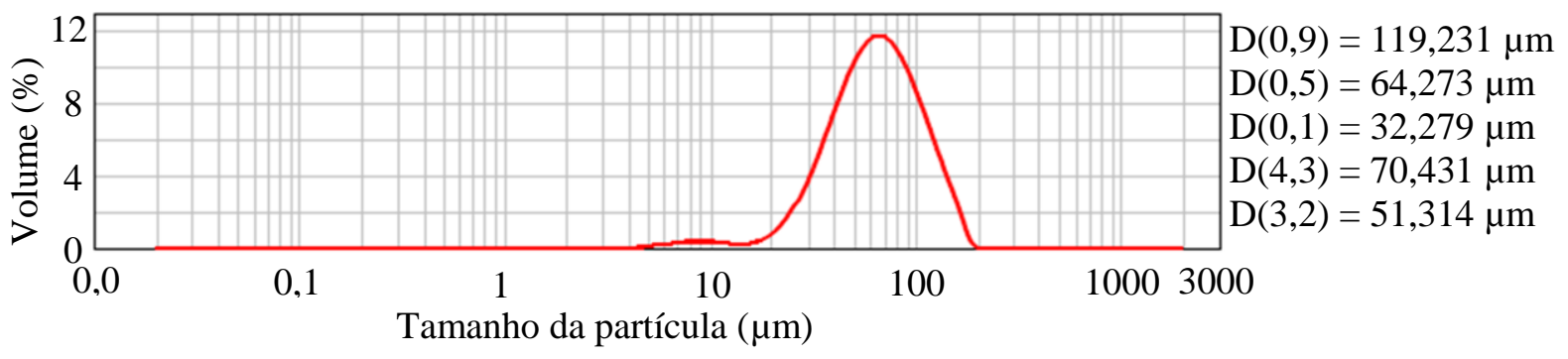

estabilizador de vórtice; e o Grupo 3 não utilizou o reforço estrutural na estrada.

O material particulado utilizado foi partículas de FCC. A Figura 6 apresenta a distribuição granulométrica das partículas, sendo que a massa específica do material é de $1400 \mathrm{~kg} / \mathrm{m}^{3}$.

\subsection{Experimentação Numérica}

A metodologia proposta para a simulação numérica consiste na aplicação de técnicas de CFD para resolver um escoamento gás-sólido com erosão.

\subsubsection{Modelagem Matemática}

A abordagem euleriana-lagrangeana com acoplamento de duas vias foi usada neste trabalho para representar o escoamento gássólido em ciclones. O campo hidrodinâmico do gás é simulado pelas equações RANS em conjunto com modelo de turbulência RSM Quadrático. As partículas sólidas são assumidas esféricas. A movimentação das partículas é analisada pelo método de rastreamento lagrangeano. A dispersão turbulenta das partículas devido à turbulência na fase gasosa foi prevista pelo modelo de trajetória estocástico DRW (Discrete Random Walk).

\subsubsection{Modelos de Erosão}

No código FLUENT (ANSYS, 2011) a erosão é exibida como fluxo de erosão e definida como a massa de material removido por unidade de área da superfície e tempo no qual as partículas sólidas atingem a superfície do material $\left(\mathrm{kg} / \mathrm{m}^{2} . \mathrm{s}\right)$.

$$
E_{R}=\sum_{p=1}^{n_{p}} \frac{\dot{m}_{p} E}{A_{\text {face }}}
$$

onde $E_{R}$ é o fluxo de erosão $\left(\mathrm{kg} / \mathrm{m}^{2} . \mathrm{s}\right), \dot{m}_{p}$ a taxa mássica de sólidos $(\mathrm{kg} / \mathrm{s}), E$ a erosão adimensionalizada e $A_{\text {face }}$ a área da face da célula $\left(\mathrm{m}^{2}\right)$. Dois modelos para o cálculo da erosão foram utilizados neste trabalho.

O modelo geral de erosão (DET NORSKE VERITAS, 2007):

$$
E=K f(\alpha) v_{p}^{n}
$$

onde $K$ é a velocidade máxima da partícula em que o limite elástico é atingido, cujo valor atribuído foi $7,48195 \times 10^{-5}(\mathrm{~m} / \mathrm{s})^{-\mathrm{n}}$ neste trabalho, $f(\alpha)$ a função do ângulo de impacto, $v_{p}$ a velocidade de impacto da partícula $(\mathrm{m} / \mathrm{s})$ e $n$ o coeficiente da velocidade da partícula, assumido como 1,2.

A função do ângulo de impacto para este modelo é dada por uma expressão polinomial:

$$
f(\alpha)=\sum_{i=1}^{n} A_{i} \alpha^{i}
$$

sendo $A_{i}$ os coeficientes do polinômio $\left(A_{1}=\right.$ $2,4647 \times 10^{-3}, A_{2}=2,9284 \times 10^{-4}$ e $A_{3}=$ $2,1974 \times 10^{-6}$ ) e $\alpha$ o ângulo de impacto.

O modelo de Oka (OKA; OKAMURA; YOSHIDA, 2005; OKA; YOSHIDA, 2005): 
Figura 7 - Variação da predição da erosão com o número de partículas computacionais.

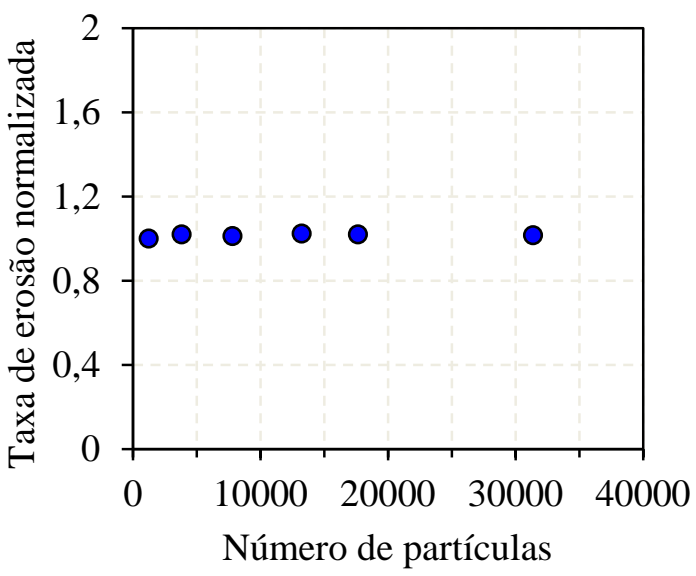

Tabela 2 - Condições de contorno.

\begin{tabular}{ll}
\hline \multicolumn{1}{c}{ Condições } & \multicolumn{1}{c}{ Configuração } \\
\hline Entrada de ar & Velocity-inlet: 25,30 e $35 \mathrm{~m} / \mathrm{s}$ \\
Saída de sólido & Pressure-outlet: $0 \mathrm{~Pa}$ \\
Saída de ar & Pressure-outlet: $0 \mathrm{~Pa}$ \\
Injeção & Material: catalisador de FCC \\
de partículas & Injeção: arquivo externo \\
& Número de partículas: 3.847 \\
& Diâmetro: $64 \mu \mathrm{m}$ \\
& Velocidade: 25,30 e $35 \mathrm{~m} / \mathrm{s}$ \\
& Taxa mássica total: $11,8 \mathrm{~g} / \mathrm{s}$ \\
& Wall: sem deslizamento \\
& Coeficiente de restituição \\
& normal e tangencial: 0,9 e 0,5 \\
\hline
\end{tabular}

$E=1 \times 10^{-9} E_{v} \rho_{w}$

$E_{v}=f(\alpha) E_{90}$

$E_{90}=L\left(H_{v}\right)^{k_{1}}\left(\frac{v_{p}}{v^{\prime}}\right)^{k_{2}}\left(\frac{d_{p}}{d^{\prime}}\right)^{k_{3}}$

Nas equações acima $E_{v}$ é a erosão volumétrica $\left(\mathrm{mm}^{3} / \mathrm{kg}\right), \rho_{w}$ a massa específica da parede $\left(2780,6 \mathrm{~kg} / \mathrm{m}^{3}\right), E_{90}$ os danos de erosão a ângulo de impacto normal $\left(\mathrm{mm}^{3} / \mathrm{kg}\right)$, $L$ constante (40), $H_{v}$ dureza de Vickers
$(0,5884 \mathrm{GPa}), k_{1}, k_{2}$ e $k_{3}$ expoentes e são $0,14,1,2$ e 0 respectivamente; $v$ ' a velocidade de referência $\left(=v_{p}\right), d^{\prime}$ o diâmetro de referência $\left(=d_{p}\right)$ e $d_{p}$ o diâmetro da partícula $(64 \mu \mathrm{m})$.

A função do ângulo de impacto é:

$f(\alpha)=(\operatorname{sen} \alpha)^{n_{1}}\left(1+H_{v}(1-\operatorname{sen} \alpha)\right)^{n_{2}}$

com $n_{1}$ e $n_{2}$ sendo 0,67 e 5,2 .

\subsubsection{Condições de Simulação}

$\mathrm{Na}$ simulação numérica, um número representativo de partículas é calculado ao invés de simular o número exato de partículas correspondente à vazão mássica. A Figura 7 mostra um estudo de independência da solução numérica com o número de partículas. A partir desse estudo é possível verificar que não existe mudança significativa na erosão com o aumento do número de partículas, a partir de aproximadamente 4000 partículas. A injeção das partículas no domínio é realizada por um arquivo externo, como condição de entrada da fase particulada.

A interação normal e tangencial entre partículas e parede foi efetuada com coeficientes de restituição de 0,9 e 0,5 , respectivamente. A massa específica e viscosidade do ar são $1,225 \mathrm{~kg} / \mathrm{m}^{3}$ e $1,789 \times 10^{-5} \mathrm{~kg} / \mathrm{m} . \mathrm{s}$; a massa específica e o diâmetro da partícula são $1400 \mathrm{~kg} / \mathrm{m}^{3}$ e 64 $\mu \mathrm{m}$.

As condições de contorno estão apresentadas na Tabela 2. O algoritmo SIMPLEC foi usado para o acoplamento pressão-velocidade. A interpolação da pressão foi calculada pelo método Presto e a interpolação do momento pelo método second order upwind. O escoamento foi transiente com $5 \mathrm{~s}$ de duração e passo de tempo igual a $0,001 \mathrm{~s}$. 
2.2.4 Quantificação da Incerteza Numérica (GCI)

\section{A técnica GCI (Grid Convergence} Index) foi utilizada para quantificação da incerteza numérica. Essa técnica é utilizada para estimar o erro de discretização em simulações de CFD foi empregada conforme recomendações de Celik et al. (2008).

Para o teste de malha, utilizaram-se três malhas distintas, com uma razão de crescimento dos elementos de $20 \%$ em todas as direções. A malha esparsa foi confeccionada com aproximadamente $224 \mathrm{mil}$ elementos, a malha intermediária com 448 mil e a malha refinada com 785 mil elementos. Os resultados analisados para pressão mostraram que o GCI da malha refinada é relativamente menor quando comparado com a malha esparsa, indicando que a dependência da simulação com o tamanho da célula foi reduzida. Tendo em vista que essa redução foi relativamente alta, a solução independente está quase alcançada, fazendo com que a malha refinada seja utilizada para as simulações numéricas deste trabalho.

\section{RESULTADOS E DISCUSSÃO}

Os Grupos 1 e 2 foram comparados entre si e o Grupo 3 foi comparado com os resultados numéricos.

\subsection{Comparação entre os Grupos 1 e 2}

Os Grupos 1 e 2 possuem reforço estrutural ao redor da região de entrada e são sem e com estabilizador de vórtice, respectivamente. Os ciclones estão enumerados de 1 a 3 , sendo 1 correspondente a menor velocidade $(25 \mathrm{~m} / \mathrm{s})$ e 3 a maior velocidade $(35 \mathrm{~m} / \mathrm{s})$. A taxa mássica de sólidos utilizada foi de 11,8 g/s. Os grupos também possuem siglas, G1 refere-se ao Grupo 1 e G2 ao Grupo 2. A Figura 8 apresenta uma imagem externa da parede oposta à entrada do ciclone. Nesta imagem pode ser observado um possível efeito do
Figura 8 - Ciclones erodidos.

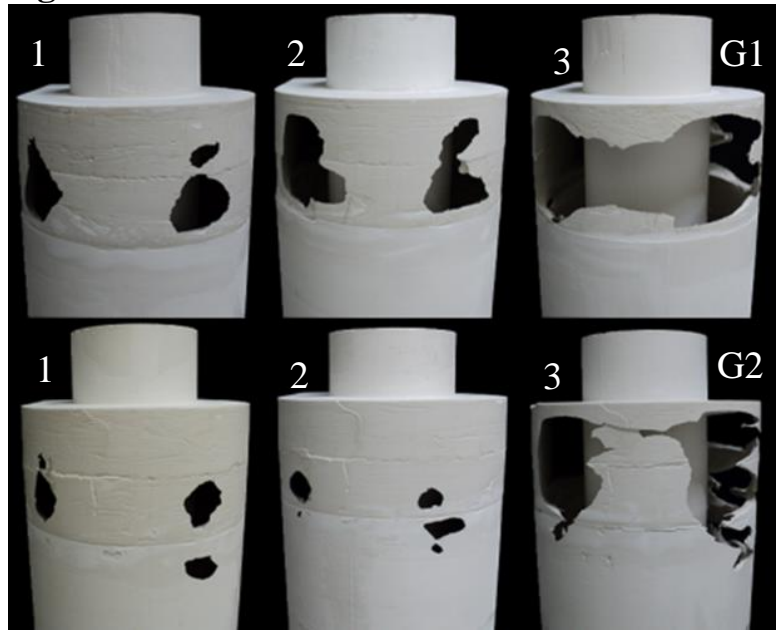

Figura 9 - Erosão na região de entrada.

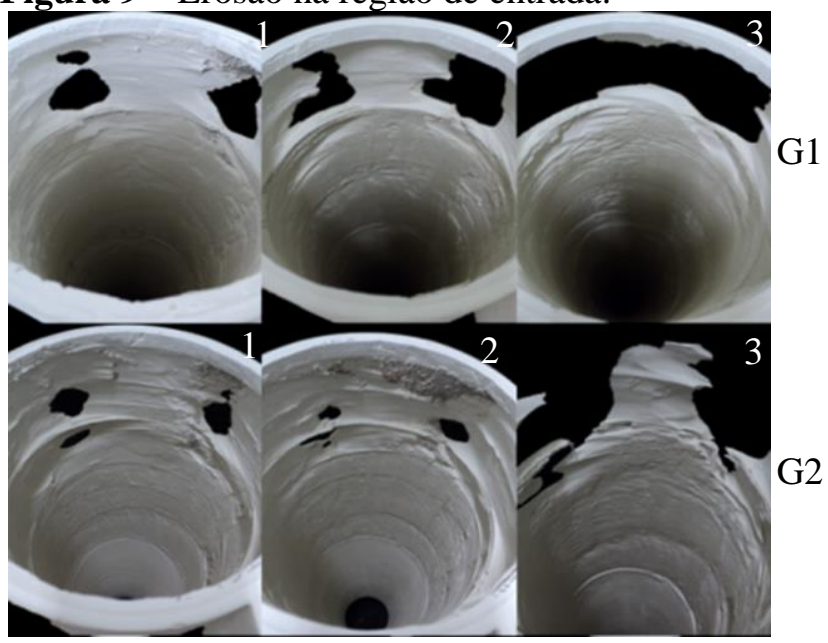

Figura 10 - Erosão no cilindro.

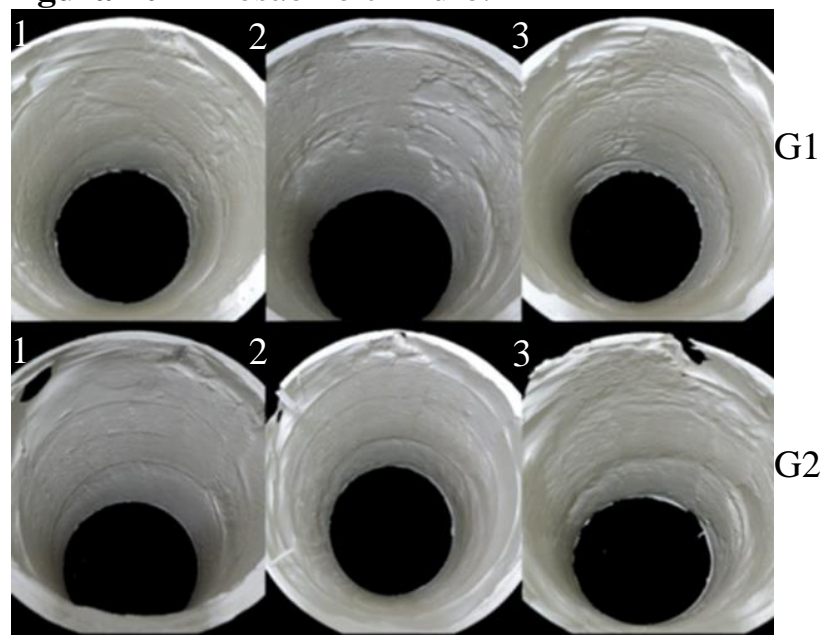

estabilizador de vórtice na redução da erosão 
Figura 11 - Erosão no cone.

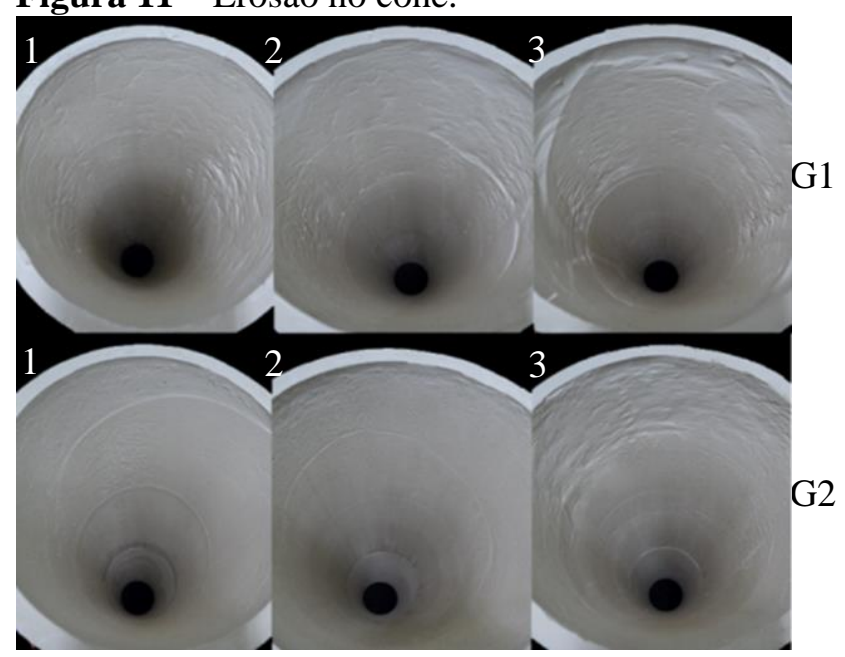

Figura 12 - Comparação da taxa de erosão global.

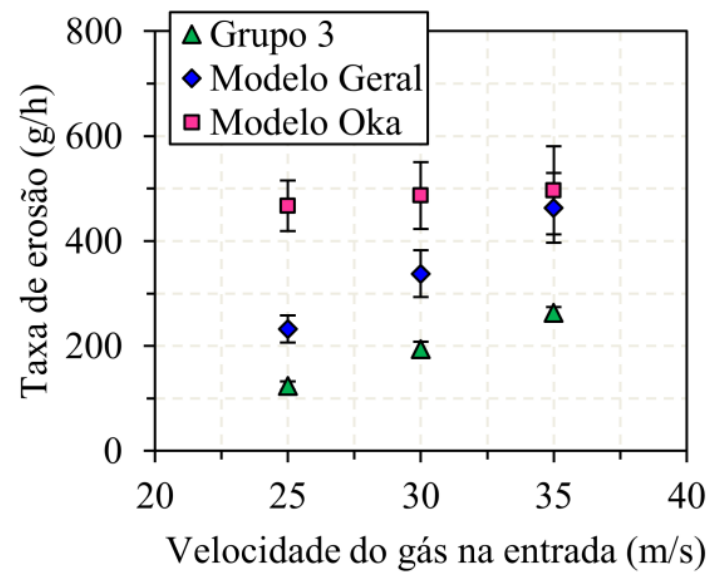

nesta região. A Figura 9 mostra a região de entrada vista pelo lado de dentro, e também é possível notar diferença na erosão quando o Grupo 1 é comparado ao Grupo 2.

$\mathrm{Na}$ parte cilíndrica, observa-se um efeito contrário, ou seja, visivelmente esta região aparenta estar mais erodida no Grupo 2 (com estabilizador de vórtice) do que no Grupo 1 (sem estabilizador de vórtice) (Figura 10).

$\mathrm{Na}$ Figura 11, nota-se novamente o efeito do estabilizador de vórtice na erosão dos cones.

\subsection{Comparação entre o Grupos 3 e as Simulações}

Figura 13 - Comparação da taxa de erosão no cone.
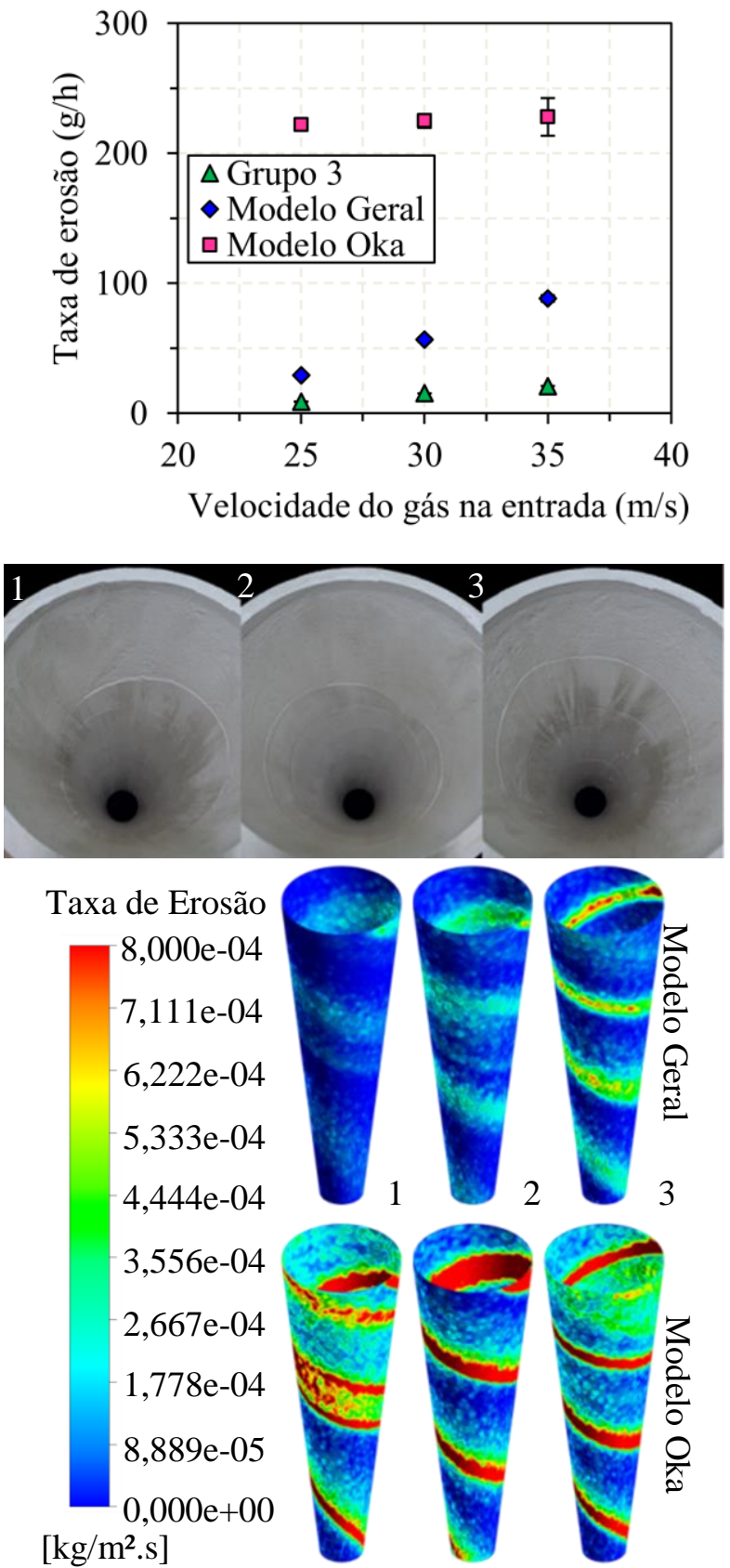

Apresentam-se nesta seção as taxas de erosão globais e por partes. O Grupo 3 foi utilizado para a comparação com os resultados numéricos. A Figura 12 apresenta a taxa de erosão global dos ciclones. Os resultados experimentais mostram que a taxa de erosão aumenta com o aumento da velocidade do gás na entrada do ciclone. Esse 
Figura 14 - Comparação da taxa de erosão no cilindro.
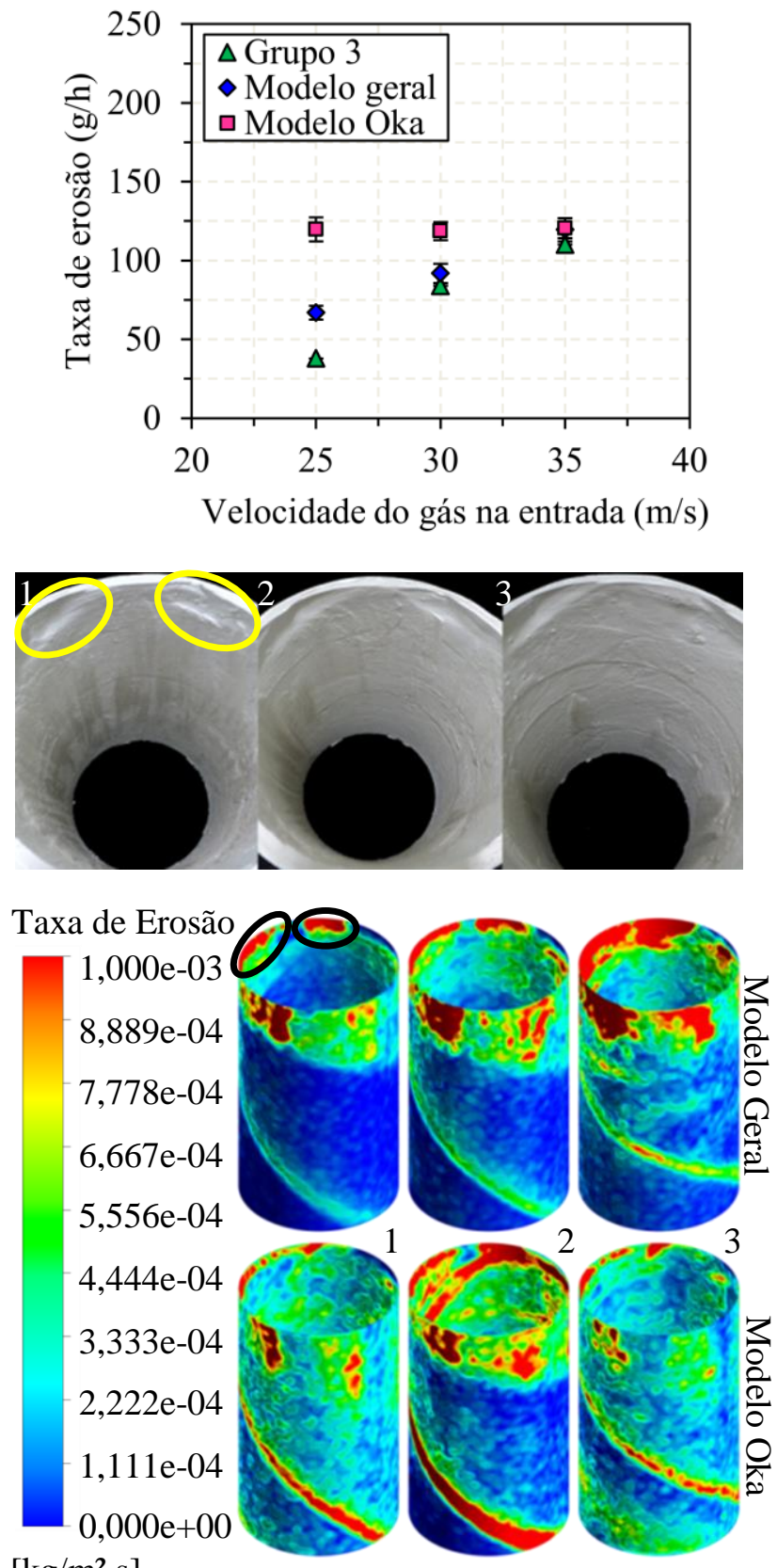

$\left[\mathrm{kg} / \mathrm{m}^{2} . \mathrm{s}\right]$

comportamento também é detectado pela simulação numérica, porém ambos os resultados numéricos superestimam a taxa de erosão. Para este caso, o modelo geral correlacionou melhor a influência da velocidade que o modelo Oka, que mantém a taxa de erosão quase constante com o aumento da velocidade. Para a erosão no
Figura 15 - Comparação da taxa de erosão na entrada.
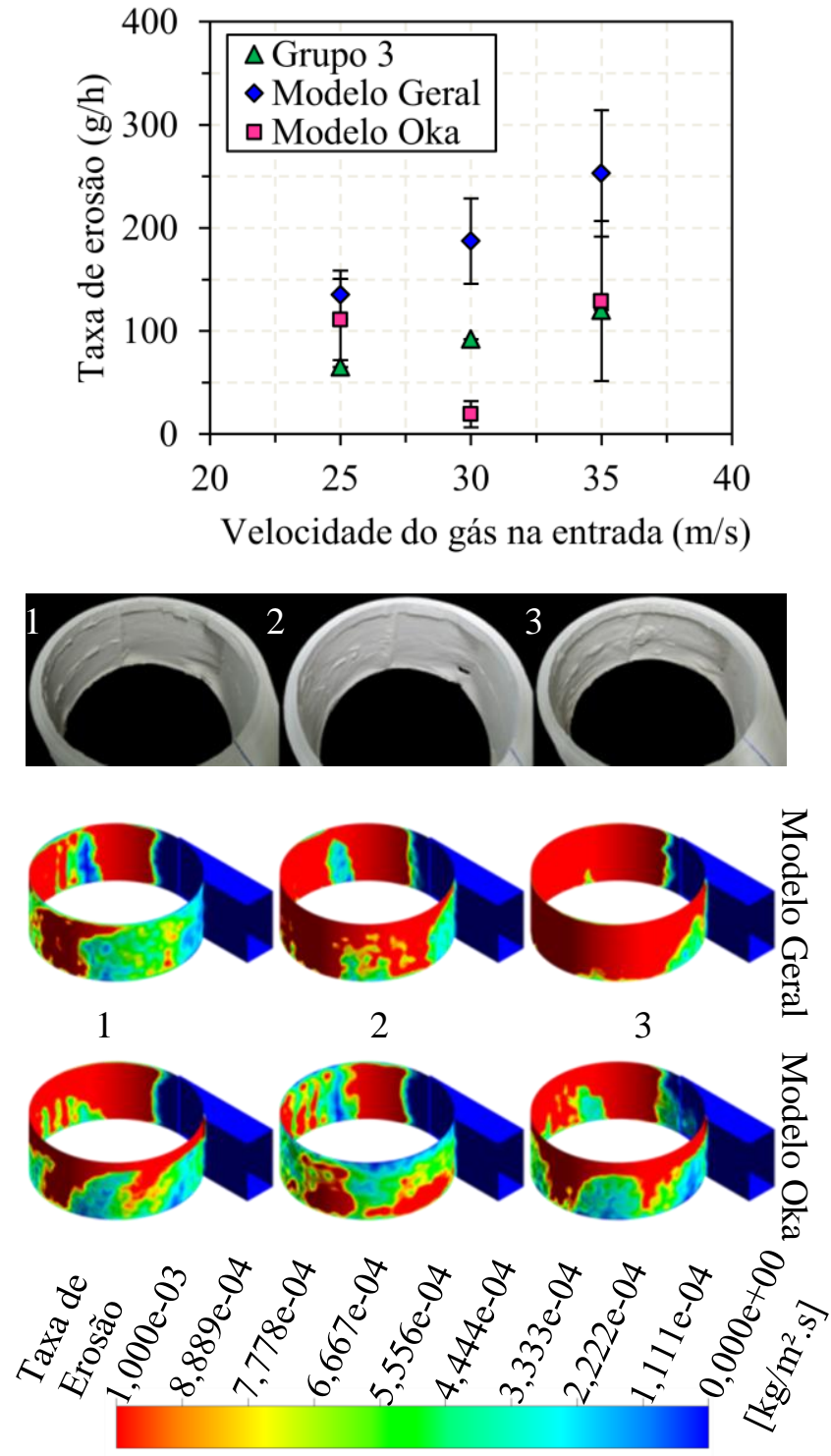

cone, nota-se que a taxa de erosão experimental é relativamente pequena. A Figura 13 exibe as taxas de erosão nos cones, que também aumenta com o aumento da velocidade, porém de maneira mais sutil. Visivelmente é difícil de afirmar o aumento da erosão. Do cone 1 para o 2, pela imagem, quase não se nota diferença, por exemplo. Observando as imagens dos cones simulados, nota-se a diferença de predição dos locais da erosão entre os modelos. O modelo Oka apresenta intensa erosão na região cônica, 
enquanto que o modelo geral apresenta uma erosão menos severa. As taxas de erosão apresentadas no gráfico também mostram que o modelo Oka superestimou a erosão no cone.

A erosão no cilindro, Figura 14, apresenta os melhores resultados preditos pelos modelos de erosão utilizados. Para as velocidades de 30 e $35 \mathrm{~m} / \mathrm{s}$ o modelo geral conseguiu predizer quantitativamente a taxa de erosão e o modelo Oka, para a velocidade de $35 \mathrm{~m} / \mathrm{s}$. Outra observação importante se deve ao fato de que o modelo geral conseguiu prever locais de erosão que são observados nos experimentos físicos, como por exemplo, as áreas que estão destacadas na Figura 14. Nota-se que pelo modelo Oka a erosão é mais distribuída ao longo de todo o cilindro.

A Figura 15 mostra as taxas de erosão para a entrada do ciclone. Curiosamente neste caso o modelo geral superestimou a erosão acima do modelo Oka, com boa predição da taxa de erosão com o modelo Oka para a velocidade de $35 \mathrm{~m} / \mathrm{s}$. Nota-se, pela imagem, diferença nos locais de erosão preditos pelos modelos de erosão. Sabe-se que o modelo Oka leva em conta os danos de erosão a ângulos de impacto normal; dessa maneira, como a região de entrada possui os maiores ângulos de impacto, estima-se que essa seja a razão pela qual a taxa de erosão calculada seja inferior à taxa de erosão calculada pelo modelo geral.

\section{CONCLUSÕES}

O estudo de erosão para diferentes velocidades e diferentes modelos de erosão em ciclones foi realizado através do software FLUENT 14.0 da ANSYS e comparado com estudos físicos. Um dispositivo para estabilização do vórtice também foi testado.

Visualmente, o estabilizador de vórtice apresentou redução da erosão, principalmente na região cônica e na região de entrada.

$\mathrm{O}$ modelo geral apresentou melhores resultados qualitativos do que o modelo Oka e em alguns casos, o modelo geral previu quantitativamente as taxas de erosão.

Em função dos resultados obtidos, supõe-se que o modelo Oka seja mais apropriado para geometrias que contenham ângulo de incidência de partículas perto de $90^{\circ}$ como, por exemplo, em curvas.

\section{NOMENCLATURA}

$\alpha \quad$ ângulo de impacto $\left[{ }^{\circ}\right]$

$A_{\text {face }} \quad$ área da face da célula $\left[\mathrm{m}^{2}\right]$

$A_{i} \quad$ constante

$b \quad$ largura da entrada [mm]

$d^{\prime} \quad$ diâmetro de referência $[\mathrm{m}]$

$d_{p} \quad$ diâmetro da partícula [m]

$D_{C} \quad$ diâmetro do cilindro [m]

$D_{l} \quad$ diâmetro da saída inferior [m]

$D_{S} \quad$ diâmetro da saída superior [m]

$E \quad$ erosão adimensionalizada [-]

$E_{R} \quad$ fluxo de erosão $\left[\mathrm{kg} / \mathrm{m}^{2} . \mathrm{s}\right]$

$E_{v} \quad$ erosão volumétrica $\left[\mathrm{mm}^{3} / \mathrm{kg}\right]$

$E_{90} \quad$ danos de erosão a ângulos de impacto normal $\left[\mathrm{mm}^{3} / \mathrm{kg}\right]$

$f(\alpha) \quad$ função do ângulo de impacto [-]

$H_{v} \quad$ dureza de Vickers [GPa]

$K \quad$ velocidade máxima da partícula em que o limite elástico é atingido [(m/s)-n]

$k_{1}, k_{2}, k_{3}$ constantes

$L \quad$ constante

$L_{C} \quad$ distância entre o final do duto e o início do cone [mm]

$L_{C O} \quad$ altura da seção cônica [mm]

$L_{e} \quad$ altura da seção de entrada [mm]

$L_{S} \quad$ altura do duto de saída [mm]

$\dot{m}_{p} \quad$ taxa mássica de sólidos $[\mathrm{kg} / \mathrm{s}]$

$n$ coeficiente da velocidade

$n_{p} \quad$ número de partículas

$n_{1}, n_{2} \quad$ constantes

$\rho_{w} \quad$ massa específica da parede $\left[\mathrm{kg} / \mathrm{m}^{3}\right]$ 
$\begin{array}{ll}v_{p} & \text { velocidade da partícula }[\mathrm{m} / \mathrm{s}] \\ v^{\prime} & \text { velocidade de referência }[\mathrm{m} / \mathrm{s}]\end{array}$

\section{REFERENCIAS}

ANSYS. FLUENT 14.0 - Theory guide. Ansys Inc, 2011.

BITTER, J. G. A. A study of erosion phenomena: Part I. Wear, v. 6, n. 1, p. 5-21, maio 1963.

CELIK, I. B; GHIA, U.; ROACHE, P. J.; FREITAS, C. J.; COLEMAN, H.; RAAD, P. E. Procedure for Estimation and Reporting of Uncertainty Due to Discretization in CFD Applications. ASME Journal of Fluids Engineering, v. 130, n. 7, 2008.

CHEN, Y.; NIESKENS, M.; KARRI, R.; KNOWLTON, T. M. Developments in cyclone technology improve FCC unit reliability. Petroleum Technology Quarterly, p. 65-71, 2010.

DENG, Y.; LIU, Y.; CHEN, Y.; ZHANG, Y. Numerical simulation of the erosion in the $90^{\circ}$ elbow. AIP Conference Proceedings, v. 1547, p. 671-683, 2013.

DET NORSKE VERITAS. Erosive wear in piping systems, 2007.

FINNIE, I. Erosion of surfaces by solid particles. Wear, v. 3, n. 2, p. 87-103, 1960.

KRAXNER, M.; PORTENKRICHNER, M.; PILLEI, $\quad$ M.; $\quad$ KOFLER, $\quad$ T.; MUSCHELKNAUTZ, U. Empirical Erosion Tests: Uniflow Cyclone as an Alternative to Reverse Flow Cyclones for a Longer Lifetime? AIChE Annual Meeting, 2013.

MAZUMDER, Q. H. Effect of liquid and gas velocities on magnitude and location of maximum erosion in u-bend. Open Journal of Fluid Dynamics, v. 02, n. 02, p. 29-34, 2012.

OKA, Y. I.; OKAMURA, K.; YOSHIDA, T. Practical estimation of erosion damage caused by solid particle impact. Part 1: Effects of impact parameters on a predictive equation. Wear, v. 259, n. 1-6, p. 95-101, 2005.

OKA, Y. I.; YOSHIDA, T. Practical estimation of erosion damage caused by solid particle impact. Part 2: Mechanical properties of materials directly associated with erosion damage. Wear, v. 259, n. 1-6, p. 102-109, jul. 2005.

PEREIRA, G. C.; SOUZA, F. J. DE; MARTINS, D. A. DE M. Numerical prediction of the erosion due to particles in elbows. Powder Technology, v. 261, p. 105117, jul. 2014.

SGROTT, O. L.; NORILER, D.; WIGGERS, V. R.; MEIER, H. F. Cyclone optimization by COMPLEX method and CFD simulation. Powder Technology, v. 277, p. 11-21, 2015.

UTIKAR, R.; DARMAWAN, N.; TADE, M.; LI, Q.; EVANS, G.; GLENNY, M.; PAREEK, V. Hydrodynamic Simulation of Cyclone Separators. In: OH, H. W. (Ed.). . Computation Fluid Dynamics. Croatia: InTech, 2010. p. 241-266.

\section{AGRADECIMENTOS}

Os autores agradecem a PETROBAS (termo cooperativo 0050.0070334.11.9) pelo suporte financeiro que permitiu a realização deste trabalho. 